\title{
Interactive Tooth Segmentation Method of Dental Model based on Geodesic
}

\author{
Shuai Yang ${ }^{1, a}$, Guanghao Jin ${ }^{1, \mathrm{~b}}$, Ruikun Wang ${ }^{1, \mathrm{c}}$ and Yongzhen $\mathrm{Ke}^{1, \mathrm{~d}}$ \\ ${ }^{1}$ School of Computer Science and Software Engineering, Tianjin Polytechnic University, Tianjin, \\ Xiqing, 300387, China \\ ayangshuai@tjpu.edu.cn, b jinguanghao@tjpu.edu.cn, cwangrk1983@163.com, \\ d keyongzhen@tjpu.edu.cn
}

\begin{abstract}
Keywords: Tooth Segmentation, Geodesic, Boundary Correction, Dental Orthopedic.
\end{abstract}
\begin{abstract}
This paper proposed an interactive tooth segmentation method based on Geodesic which aims to solve the inaccurate problem of segmentation in dental model for irregular teeth case. The features of the teeth are automatically acquired according to the prior knowledge. Then based on those features, the boundary of each tooth is obtained by the improved regional growth method and corrected by combining the correction points from the user inputting based on the shortest geodesic path, so that, the teeth are precisely segmented according to the boundary. The experimental results show that our method can be used to segment different kinds of teeth correctly and the computational speed and the precision of segmentation are improved obviously. Furthermore, as the features and the initial boundaries can be acquired automatically, the complexity of the implementation is significantly reduced.
\end{abstract}

\section{Introduction}

With the improvement of life quality, more and more people pay attention to the healthcare of their tooth as it affects the processes of appearance and digestion. Brace is one of the effective equipment that is used in dental orthopedic. Currently, the metal brace is widely used but is not beautiful and difficult to install make more and more people start to use hidden brace instead. To make the hidden brace, the preparation process should contain the following steps: scanning the teeth to get the dental model, teeth segmentation, fitting dental arch line and simulating the orthodontic process. The dental model can be easily obtained by a three-dimensional scanner. The segmentation step can affect the correctness of the following step of making the invisible brace and the accuracy is very important. Thus, we focus on the segmentation which is the key factor of the whole process to improve both efficiency and accuracy.

In recent years, numerous tooth segmentation approaches have been proposed. The most recent works include semi-automatic ${ }^{[1,8]}$, automated method ${ }^{[2,3,5]}$ and morphology ${ }^{[6]}$. The automatic methods cannot reflect the subjective idea of the dentist, and lack adaptability to the complex and varied dental model. The semi-automatic methods take user interaction as a way of selecting seeds which is the initial condition of segmentation, and it does not optimize the results, so cannot achieve high segmentation accuracy. There is a threshold in morphology method, and appropriate threshold is difficult to be obtained, and the method is high time complexity.

Zhang, Chi, et al ${ }^{[3]}$ proposed an interactive marker-controlled algorithm which is based on the watershed algorithm. In this algorithm, the artificial markers are used as the seeds for the segmentation. Each seed is a slice that can be used to control the segmentation process to improve accuracy. This algorithm follows the minima rule that is proposed by Hoffman et al and expands region based on Fast Marching Watershed to segment a teeth from dental mold.

In papers [7], a method of interactive tooth segmentation is proposed. The method requires the user to select any number of boundary control points on the dental model. It uses a shortest path to connect the given control point and determine the final split boundary. Then, it uses the given seed points from the target tooth to start the process of "flooding" to get the target area of segmentation. 
Although this method has good applicability and is easy to be implemented,but the user should be experienced and need to operate many times which makes it consumes much time. The result depends on the experience of user operation and some other factors like the skills of the seeds selection.

Those methods have the problem of accuracy or efficiency of segmentation in irregular tooth case. Therefore, an interactive tooth segmentation method based on geodesic was proposed. Our method corrects the original tooth boundary according to user interaction which makes it possible to get higher segmentation efficiency to meet the needs of dental orthodontics. In more details, our method is based on the interactive mark control algorithm ${ }^{[3]}$ and the shortest geodesic path. The improvements contains three aspects: firstly, the dental model is refined and smoothed and we cancel the sorting operation which is time consuming. Furthermore, the useless data is deleted and the noise is decreased, so that, the segmentation efficiency and precision is improved. Secondly, to improve the precision of the boundary of segmentation, our method interactively correct the teeth boundary based on geodesic path. Thirdly, the seeds for obtaining the tooth boundary is extracted automatically to reduce the interaction times, will be used to improve the accuracy of the separation.

\section{Proposed Method}

In order to improve the segmentation speed and accuracy, we propose the tooth segmentation algorithm with the following process as shown in Fig. 1.

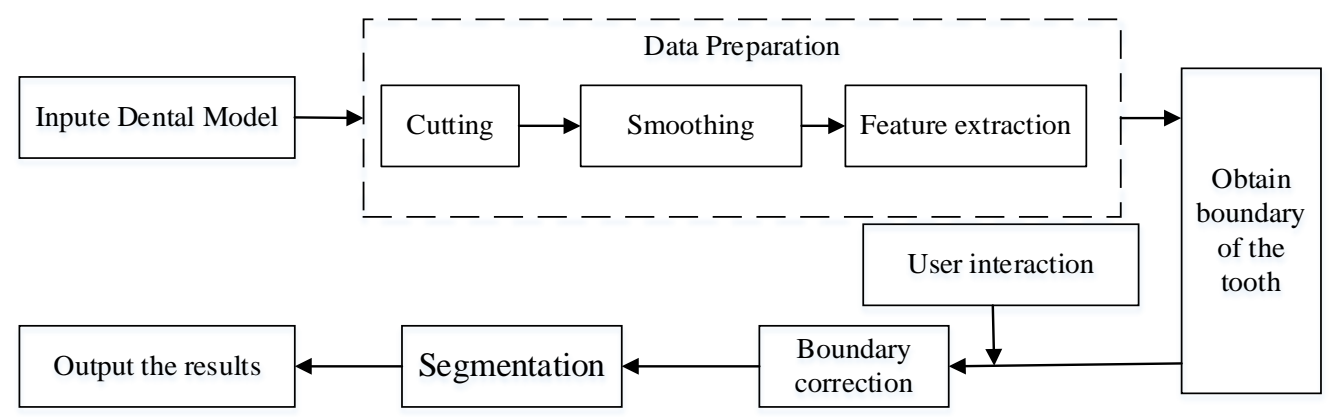

Fig. 1 The Proposed Algorithm

The process of the proposed algorithm. Our interactive tooth segmentation method based on geodesic path is as below:

(1) Data preparation: It uses triangle mesh to represent all the teeth. Then, it smooths the mesh to decrease noises of the data and cuts the dental model to reduce the number of triangles, so that, the segmentation efficiency can be improved. The features of the tooth are extracted as the seeds of the initial condition of segmentation.

(2) Obtaining tooth boundary: It accesses to the stack which stores the numbers of the mesh triangles and separate those triangles to several groups based on the minima rule ${ }^{[11]}$, then extract the boundary.

(3) Interactive correction and segmentation: The tooth boundary is corrected by user interaction based on shortest geodesic path. According to the boundary single teeth can be segmented.

Interactive tooth segmentation method based on geodesic path is shown in Fig. 1.

Data Preparation. Data preparation consists of three steps: model cutting, mesh smoothing, and feature extraction.

The main purpose of model cutting is to reduce the number of triangles of the mesh for the following processes. The cutting section uses a plane in a three-dimensional space to cut the model so that the original input data is divided into two parts, the portion containing the tooth data and the useless data near the bottom of the model. Another part of the data preprocessing job is mesh smoothing. In order to reduce the noise and improve the precision of the segmentation, the Laplacian operator is used to smooth the mesh. Features extracting uses the method proposed by Kumar et al ${ }^{[4]}$. The method uses the local extremum as the features in the dental model. Then, we regroup the features by the following steps. We first find a dental arch curve for a given feature. Then, along the 
curve, a series of partitions can be detected. Those partitions devide the features into different groups which ensure the same features of each tooth can be in the same stack. Fig.2 (b) shows the tooth features grouping, in which red and green marks the different groups of features.

Obtain boundary of the tooth. Based on the traditional interactive marking algorithm for the segmentation, the triangles are initially grouped and the boundary of the teeth is detected. Different from the traditional marking algorithm, our method uses a set of markers obtained in the data preparation process. The number of features is related to the types of teeth, so that, the number of markers is also different in marking algorithm. More markers do not increase the complexity of the algorithm, but can improve the accuracy of segmentation, and can avoid the over-segmentation and under-segmentation problems. More details and analysis can be seen in section "Experiments and Results".

To obtain the tooth boundary, the triangles that have been marked by a same label can be seen as a teeth area. The area plays an important role when it calculates the teeth boundary. It uses three-tuples ( $\mathrm{f}, \mathrm{m}, \mathrm{c}$ ), where $\mathrm{f}$ is the face of the mesh, $\mathrm{m}$ is the marker, c represents the curvature of two adjacent faces of the mesh. Our algorithm first pushes all of the marked faces and their adjacent faces to the stack. A maximum curvature that has not been accessed is taken out from the stack with the progress of iteration. Pseudo code of the algorithm is shown in Table 1. The original tooth boundary is shown in Fig. 2 (c).

Table 1 The pseudo-code for obtaining the original tooth boundary

\begin{tabular}{|c|c|}
\hline seudo-code of obtaining the original tooth bou & $:\left\{E_{k}\right\}$-each tooth edge \\
\hline Input: mesh $\mathrm{M}$ & \\
\hline //Initialize tag of each face & //Tag each face one by one \\
\hline Foreach face $\mathrm{f}_{\mathrm{i}}$ of mesh $\mathrm{M}$ do & While $S$ is not empty do \\
\hline $\begin{array}{l}\text { If } \mathrm{f}_{\mathrm{i}} \text { belongs to marker } \mathrm{m}_{\mathrm{k}} \text { Then } \mathrm{f}_{\mathrm{i}} \cdot \mathrm{tag}=\mathrm{m}_{\mathrm{k}} \\
\text { Else } \mathrm{f}_{\mathrm{i}} \text { tag=null }\end{array}$ & $\begin{array}{l}\left(\mathrm{f}_{\mathrm{i}}, \mathrm{m}_{\mathrm{k}}, \mathrm{c}_{\max }\right)=\text { Find maxinum } \mathrm{c}_{\max } \text { in } \mathrm{S} \\
\mathrm{V}_{\mathrm{i}} \text { tag }=\mathrm{m}_{\mathrm{r}}\end{array}$ \\
\hline End for & $\mathrm{S}=\mathrm{S}-\left\{\left(\mathrm{f}_{\mathrm{j}}, \mathrm{m}_{\mathrm{k}}, \mathrm{C}_{\max }\right)\right\}$ \\
\hline //Initialize stack S(f, m, c) & Foreach neighboring face $f_{j}$ of $v_{j}$ do \\
\hline$S=\{\}$ & If $\mathrm{v}_{\mathrm{j}} \cdot \operatorname{tag}=$ null then $\mathrm{S}=\mathrm{S} \cup\left\{\left(\mathrm{f}_{\mathrm{j}}, \mathrm{m}_{\mathrm{k}}, \mathrm{c}_{\mathrm{ij}}\right)\right\}$ \\
\hline Foreach marker $m_{k}$ do & End for \\
\hline Foreach face $f_{i}$ of $m_{k}$ do & End while \\
\hline $\begin{array}{l}\text { Foreach neighboring face } \mathrm{f}_{\mathrm{j}} \text { of } \mathrm{f}_{\mathrm{i}} \text { do } \\
\quad \text { If } \mathrm{f}_{\mathrm{j}} \text {.tag=null then } \mathrm{S}=\mathrm{S} \cup\left\{\left(\mathrm{f}_{\mathrm{j}}, \mathrm{m}_{\mathrm{k}}, \mathrm{c}_{\mathrm{ij}}\right)\right\}\end{array}$ & $\begin{array}{l}\text { Foreach }\left(\mathrm{f}_{\mathrm{a}}, \mathrm{m}_{\mathrm{i}}, \mathrm{c}_{\mathrm{a}}\right) \text { do } \\
\quad \text { If } \mathrm{i}=\mathrm{k} \text { then mesh } \mathrm{M}_{\mathrm{k}}=\mathrm{M}_{\mathrm{k}} \cup\left\{\mathrm{f}_{\mathrm{a}}\right\}\end{array}$ \\
\hline End for & End for \\
\hline End for & Find all Edges $\left\{E_{k}\right\}$ of $\left\{M_{k}\right\}$ \\
\hline End for & Return $\left\{\mathrm{E}_{\mathrm{k}}\right\}$ \\
\hline
\end{tabular}

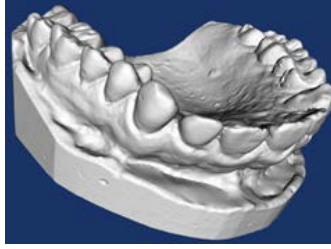

(a) Original dental model

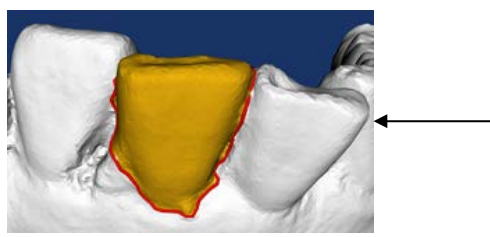

(f) The separation result

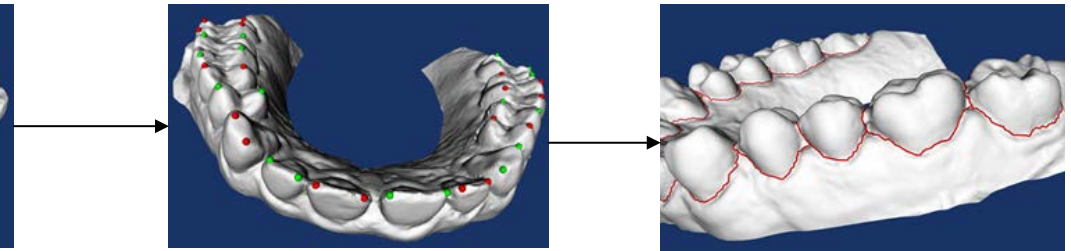

(b) The result of data preparation

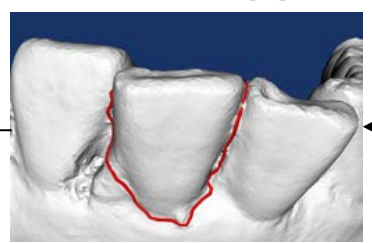

(e) The corrected boundary

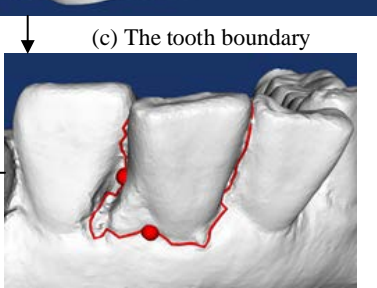

(d) The markers for correction

Fig. 2 The output of each step of the algorithm 
Interactive correction for the boundary based on the shortest geodesic path. Based on the user interaction, it modifies the boundary points to get a better tooth boundary. Obviously, the acquired original boundary substantially is located near the junction of the teeth and the gums. When the teeth and gums has no obvious boundary or there are foreign bodies, the acquired boundary is imperfect as shown in Fig. 2 (d). The boundary is optimized by following steps. First, the user selects two points of the boundary, one as the starting point, the other as the end point (Fig. 2 (d)). Then, our method calculates a shortest geodesic path $\mathrm{L}_{s}$ from the origin to the destination. The original boundary of the teeth is divided into two parts. We name the longer portion of boundary as $\mathrm{L}_{\mathrm{p}}$, the correction is $\mathrm{L}_{\mathrm{s}}+$ $\mathrm{L}$ p. Pseudo code is shown in Table 1 which can explain the whole process, the corrected boundary as shown in Fig. 2 (e), the segmentation results as shown in Fig. 2 (f).

We regard the triangle meshes as a weighted graph, in which the triangles vertex is the vertex of the graph, the triangles edge as the edge of the graph, and the weight is the length of the triangles edge. The shortest geodesic path between two points in mesh can be calculated by Dijkstra algorithm.

\section{Experiments and Results}

The experiments are to verify the accuracy and efficiency of the proposed algorithm. The dental model is constructed by a three-dimensional scanner. The information of the computational resource is as below: processor Intel (R) Core (TM) i7-4790CPU @ 3.6GHz, memory 8GB, 64 bit Windows10 System platform. The result of each step of the algorithm is shown in Fig. 2, and the segmentation results is shown in Fig. 3.

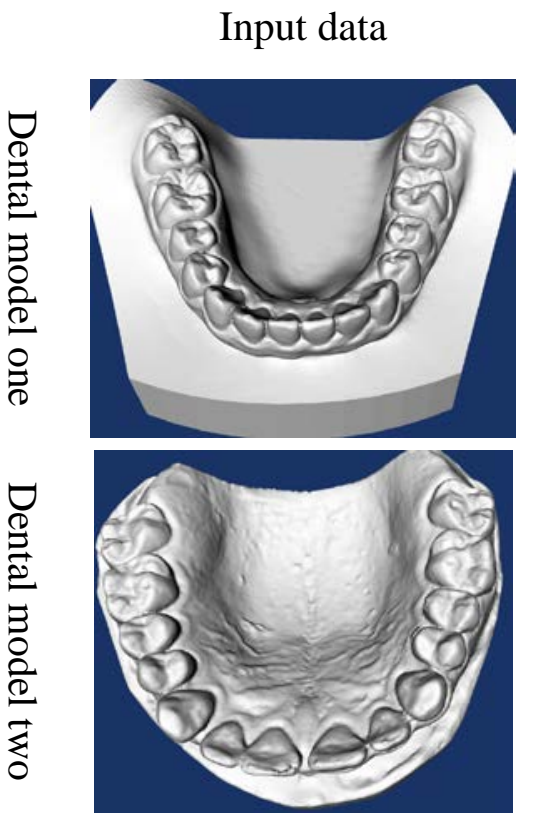

Segmentation results
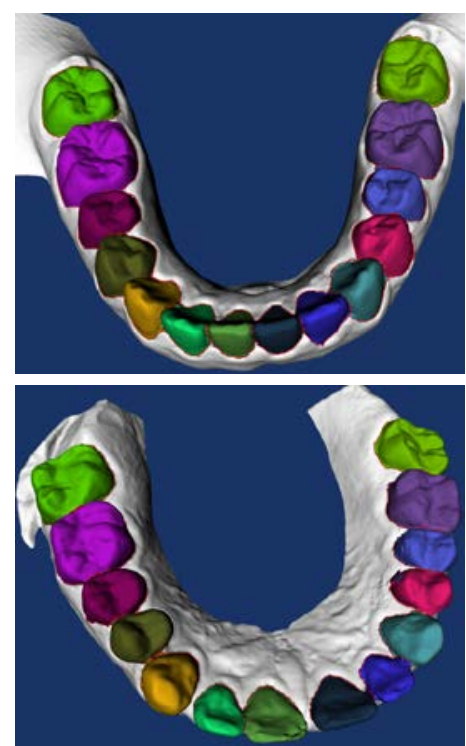

Segmentation results
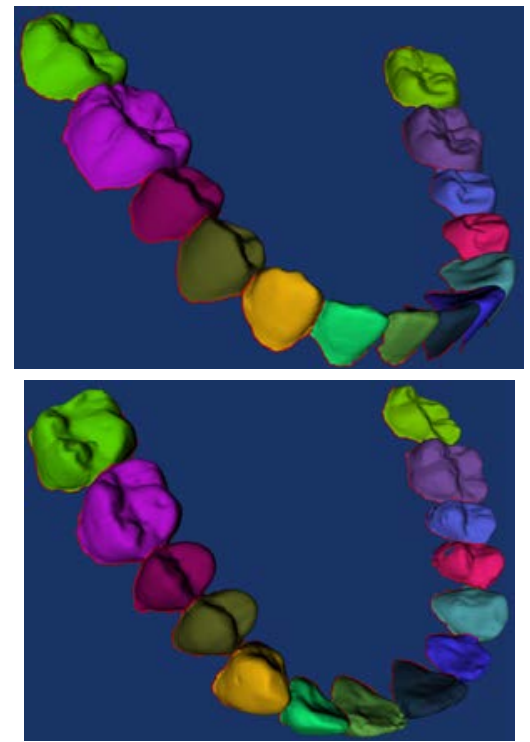

Fig. 3 The segmentation results of our algorithm

In this paper, the model is initially cut into two parts, which can reduce the number of triangles and it effectively avoids the sorting operation of the data in the stack. These revisions can improve the efficiency of the algorithm. The time comparison of our method and the the algorithm as paper [3] is shown in Table 2. Experimental data shows that the speed of our proposed method is about 6 times faster than the algorithm in paper [3].

Table 2 Algorithm time comparison

\begin{tabular}{lcccc}
\hline & $\begin{array}{c}\text { Number of } \\
\text { triangles }\end{array}$ & $\begin{array}{c}\text { Number of } \\
\text { vertices }\end{array}$ & $\begin{array}{c}\text { The algorithm of } \\
\text { paper[3](s) }\end{array}$ & $\begin{array}{c}\text { Our algorithm } \\
(\mathrm{s})\end{array}$ \\
\hline Model one & 70942 & 35473 & 88 & 13 \\
Model two & 226843 & 113489 & 368 & 59 \\
\hline
\end{tabular}

Paper [3] algorithm only uses interaction inputting points as the markers which easily lead to the failures of obtaining tooth boundaries (Fig. 4 (b)). Our algorithm using the cutting boundary points as 
the markers and the feature points are also applied as the markers, so that, our method can effectively acquire the boundaries that can avoid the failures (Fig. 4 (a)).

In the paper, we use the extracted tooth features as the markers to obtain the better boundary, which makes the algorithm result more stable and correct. The algorithm has a set of features of tooth as the markers and those markers substantially fixed at a limited area, avoiding the problem of paper [3] in obtaining boundaries. Experimental results shown in Fig. 5.

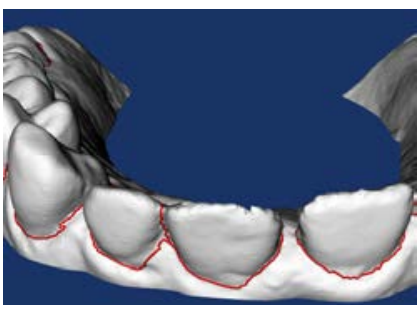

(a) Our method

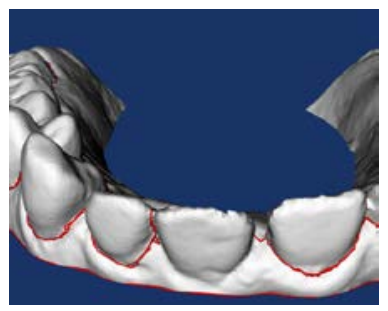

(b) The method of [3]

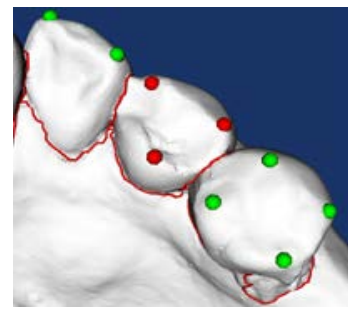

(a) Our method

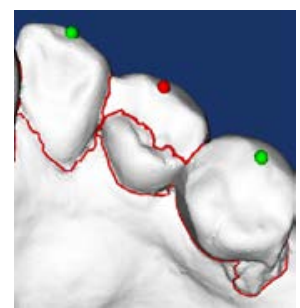

(b) The method of [3]

Fig. 4 The effect of cutting boundary on obtaining Fig 5. The effect of markers on obtaining the the original tooth boundary.

\section{Conclusions}

This paper presents an interactive tooth segmentation algorithm based on the geodesic path. The experimental results show that the proposed algorithm is faster than the traditional interactive algorithm in obtainning tooth boundary, and the segmentation accuracy is obviously improved. Of course, the algorithm also has some limitations such as noise-sensitive.

\section{Acknowledgments}

This research was partially supported by the Science and Technology Major Projects of Tianjin, China (Grant No. 15ZXHLGX00300).

\section{References}

[1] Kronfeld, Thomas, D. Brunner, and G. Brunnett. "Snake-Based Segmentation of Teeth from Virtual Dental Casts." Computer-Aided Design and Applications 7.2(2010):221-233.

[2] Kumar, Yokesh, et al. "Improved Segmentation of Teeth in Dental Models." Computer-Aided Design and Applications 8.2(2011):211-224.

[3] Zhang, Chi, et al. Marker-Controlled Perception-Based Mesh Segmentation. Marker-controlled perception-based mesh segmentation. 2004:390-393.

[4] Kumar, Yokesh, R. Janardan, and B. Larson. "Automatic Feature Identification in Dental Meshes." Computer-Aided Design and Applications 9.6(2013):747-769.

[5] Li Z, and Wang H. "Interactive Tooth Separation from Dental Model Using Segmentation Field." Plos One 11.8(2016):e0161159.

[6] Wu, Kan, et al. "Special Section on CAD/Graphics 2013: Tooth segmentation on dental meshes using morphologic skeleton." Computers \& Graphics 38.1(2014):199-211.

[7] Sinthanayothin, C, and W. Tharanont. "Orthodontics treatment simulation by teeth segmentation and setup." International Conference on Electrical Engineering/electronics, Computer, Telecommunications and Information Technology, 2008. Ecti-Con IEEE, 2008:81-84.

[8] Liao, S. H., et al. "Automatic Tooth Segmentation of Dental Mesh Based on Harmonic Fields." Biomed Research International 2015.3(2015):1-10. 